\title{
ENUMERATING 2-CELL IMBEDDINGS OF CONNECTED GRAPHS
}

\author{
BRUCE P. MULL, ROBERT G. RIEPER AND ARTHUR T. WHITE
}

(Communicated by Thomas H. Brylawski)

\begin{abstract}
A systematic approach is developed for enumerating congruence classes of 2-cell imbeddings of connected graphs on closed orientable 2-manifolds. The method is applied to the wheel graphs and to the complete graphs. Congruence class genus polynomials and congruence class imbedding polynomials are introduced, to summarize important information refining the enumeration.
\end{abstract}

1. Introduction. An imbedding of a graph $G$ into a closed orientable 2-manifold $S$ is a homeomorphism $i: G \rightarrow S$ of $G$ (as a one-dimensional simplicial complex in $R^{3}$ ) into $S$. If every component of $S-i(G)$ is a 2-cell, then $i$ is said to be a 2-cell imbedding. (This requires that $G$ be connected.) If we fix an orientation on $S$ (say clockwise), then $i$ is an oriented imbedding.

Gross and Furst [3] have issued a call-to-arms on enumerative methods in topological graph theory, and this paper is in response to that call. We wish to count equivalence classes of 2-cell imbeddings under a natural equivalence relation which regards as identical two imbeddings which "look alike." To this end, the oriented imbeddings $i: G \rightarrow S$ and $j: G \rightarrow S$ are said to be congruent if there exists an isomorphism of the labeled image graphs $i(G)$ and $j(G)$ that can be extended to an orientation-preserving autohomeomorphism of the surface $S$. The equivalence classes this gives rise to are more readily enumerated in their guise as algebraic equivalence classes of graph rotations, and this is the approach we take here.

Let $G$ be a connected graph, with vertex set $V(G)$ and edge set $E(G)$. The set of neighbors of $v \in V(G)$ is given by $N(v)=\{u \in V(G) \mid u v \in E(G)\}$. A rotation at $v$ is a cyclic permutation $\rho_{v}: N(v) \rightarrow N(v)$, and a set $\rho=\left\{\rho_{v}\right\}_{v \in V(G)}$ is called a rotation on $G$. A map is a pair $M=(G, \rho)$, and it is well known (see, for example, Chapter 6 of [5]) that $M$ determines a 2-cell imbedding $i: G \rightarrow S_{k}$ into a closed orientable 2-manifold $S_{k}$ of genus $k$, where $k$ is readily determined from $\rho$; conversely, every such imbedding of $G$ has a corresponding $\rho$ associated with it.

Form the set $R(G)$ of all rotations of $G$; it is easy to see that $|R(G)|$ $=\prod_{v \in V(G)}(d(v)-1)$ !, where $d(v)$ is the degree of vertex $v$. Some pairs of these

Received by the editors March 1, 1987. Bruce P. Mull presented the contents of this paper, on April 11, 1987, to the Eleventh Midwest Graph Theory Conference, Eastern Michigan University, Ypsilanti, Michigan.

1980 Mathematics Subject Classification (1985 Revision). Primary 05C10; Secondary 05C25.

Key words and phrases. Topological graph theory, connected graph, closed orientable 2-manifold, 2-cell imbedding, congruent imbeddings, rotation, equivalent rotations, wheel graph, complete graph, congruence class genus polynomial, congruence class imbedding polynomial, graph automorphism, map automorphism. 


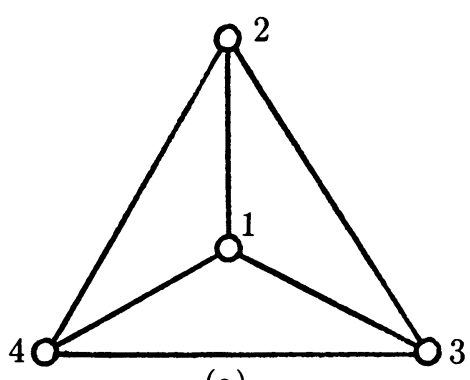

(a)
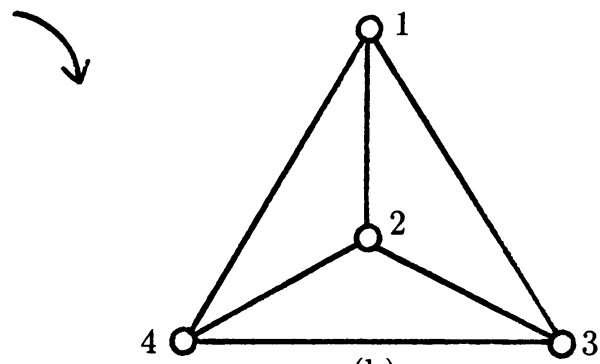

(b)

FIGURE 1

rotations give rise to maps (that is, 2-cell imbeddings) which are clearly different: perhaps the genera differ, or the sizes of the regions differ where the genera agree. Other differences may be more subtle. Or perhaps the two maps are essentially alike: one is merely a relabelling of the other. The equivalence relation on $R(G)$ which makes this distinction is defined as follows. Two rotations $\rho, \sigma \in R(G)$ are equivalent if there is an automorphism $\alpha \in \operatorname{Aut} G$ so that $\alpha \rho_{v} \alpha^{-1}=\sigma_{\alpha(v)}$, for all $v \in V(G)$; the common domain for these two permutations is $\alpha(N(v))=N(\alpha(v))$. (This familiar variety of algebraic equivalence was previously studied for graph rotations by Biggs [1]; see also Chapter 5 of Biggs and White [2].) Then a map automorphism for $M=(G, \rho)$ is a graph automorphism for $G$ giving $\rho$ equivalent to itself, and Aut $M=\operatorname{Aut}(G, \rho)=\left\{\alpha \in \operatorname{Aut} G \mid \alpha \rho_{v} \alpha^{-1}=\rho_{\alpha(v)}, \forall v \in V(G)\right\}$. We observe that such an $\alpha$ preserves adjacency (as a graph automorphism) and oriented region boundaries (as a map automorphism).

Our task is to count the set of equivalence classes of rotations for $G$; that is, the set $C(G)$ of congruence classes of imbeddings of $G$. (Thus, the equivalence of two rotations for $G$ induces a congruence between the corresponding imbeddings for which the isomorphism of labelled image graphs preserves labels and the autohomeomorphism of the ambient surface is orientation preserving.) To apply the permutation group theory of the next section, we regard Aut $G$ as acting on $R(G)$ as follows: let $\rho=\left\{\rho_{v}\right\}_{v \in V(G)} \in R(G)$, and $\alpha \in$ Aut $G$; then

$$
\alpha(\rho)=\left\{\alpha \rho_{v} \alpha^{-1}\right\}_{v \in V(G)}=\left\{\sigma_{\alpha(v)}\right\}_{v \in V(G)}=\left\{\sigma_{v}\right\}_{v \in V(G)},
$$

for some $\sigma \in R(G)$.

For example, if $M$ is the map $\left(K_{4}, \rho\right)$ of Figure 1a $\left(\rho_{1}=(2,3,4) ; \rho_{2}=(1,4,3)\right.$; $\left.\rho_{3}=(1,2,4) ; \rho_{4}=(1,3,2)\right)$ and if we take $\alpha=(1,2) \in$ Aut $K_{4}$, then $\alpha(\rho)=$ $\left\{\sigma_{1}, \sigma_{2}, \sigma_{3}, \sigma_{4}\right\}$, where $\sigma_{1}=(2,4,3), \sigma_{2}=(1,3,4), \sigma_{3}=(1,4,2), \sigma_{4}=(1,2,3)$. The resulting map $\left(K_{4}, \sigma\right)$ see Figure $1 \mathrm{~b}$ is the "mirror image" of $\left(K_{4}, \rho\right)$, and the two maps are equivalent (and the corresponding imbeddings are congruent) under $\alpha$.

2. The basic tool. As indicated above, we let Aut $G$ act on the set $R(G)$ of rotations for $G$. Fix $\rho \in R(G)$, and let $M=(G, \rho)$.

THEOREM 1. The number of rotations of $G$ equivalent to $\rho$ is the index [Aut $G:$ Aut $M$ ]. 
Proof. It is well known (see Chapter 1 of [2], for example) that the size of the orbit of an object under the action of a permutation group is the index, in the group, of the stabilizer of the object.

THEOREM 2. The number of equivalence classes of maps $M=(G, \rho)$ having $G$ as the underlying graph is

$$
|C(G)|=\frac{1}{|\operatorname{Aut} G|} \sum_{\alpha \in \operatorname{Aut} G}|F(\alpha)|,
$$

where $F(\alpha)=\{\rho \in R(G) \mid \alpha(\rho)=\rho\}$ is the fixed set of $\alpha$.

PROOF. This is just Burnside's Lemma for the present context.

We illustrate these two theorems for $G=K_{4}$ (continuing the example of the previous section; in fact, we sustain this example throughout this paper. The calculations and the figures below also appear in [2]). We first observe that $\left|R\left(K_{4}\right)\right|=2^{4}=$ 16. For $M=\left(K_{4}, \rho\right)$ as given in Figure 1a, we find Aut $M=A_{4}$. Since Aut $K_{4}=S_{4}$ (the symmetric group of degree four), there are by Theorem $1\left[S_{4}: A_{4}\right]=2$ maps in this congruence class. The other one is the "mirror image", as shown in Figure $1 b$.

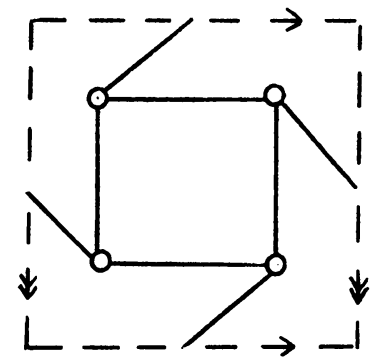

(a)

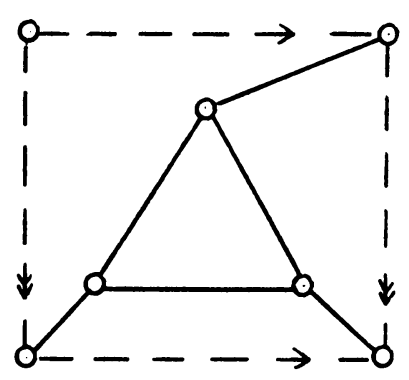

(b)

\section{FIGURE 2}

To classify all sixteen rotations for $K_{4}$ we note that $|F(\alpha)|$ is constant on each conjugacy class of $S_{4}$; we obtain, by ad hoc methods for the time being, Table 1. (Powerful general methods appear in §3.)

\section{TABLE 1}

$\begin{array}{lccccc}\text { Class representative }(\alpha): & e & (12) & (123) & (12)(34) & (1234) \\ \text { Number in class: } & 1 & 6 & 8 & 3 & 6 \\ |F(\alpha)|: & 16 & 0 & 4 & 4 & 2\end{array}$

By Theorem 2 there are $(16+0+32+12+12) / 24=3$ congruence classes of maps. One of these is spherical, as already noted (Figure 1); the other two are toroidal see Figure 2. In Figure 2a the torus is divided into a square and an octagon, with Aut $M=Z_{4}$; thus there are $24 / 4=6$ maps in this class. Figure $2 \mathrm{~b}$ divides the torus into a triangle and a nonagon, with Aut $M=Z_{3}$; thus there are $24 / 3=8$ maps in this class. 
Looking at Table 1 , we observe the following:

(i) If an automorphism of $K_{4}$ fixes more than one vertex, then it fixes no rotations unless it is the identity--in which case it fixes them all.

(ii) Each graph automorphism which fixes at least one rotation either has all cycles or all but one of uniform length, and in the latter case the exception is a singleton (corresponding to the fixed vertex).

These two properties generalize, as we establish in the next section.

3. Main results. The determination of $|C(G)|$ using Theorem 2 is greatly simplified with the following observations. First, we need not consider each permutation in Aut $G$ individually, since $|F(\alpha)|$ is constant on every conjugacy class, and in many cases is in fact zero. Second, the value of $|F(\alpha)|$ is readily computable from the cycle type of the permutation $\alpha$. These observations are formalized in the theorems below.

THEOREM 3. If $\alpha \in$ Aut $G$ fixes two adjacent vertices, then either $\alpha$ is the identity permutation or $|F(\alpha)|=0$.

Proof. This is Lemma 5.2.5 of [2].

For $\alpha \in$ Aut $G$ and $v \in V(G)$ define the fixed set at $v$ to be the set of rotations at $v$ fixed by $\alpha$ under conjugation (the set may be empty). Denote the set by $F_{v}(\alpha)$. In the disjoint cycle representation of $\alpha$, let $l(v)$ be the length of the cycle containing $v$; i.e. $l(v)$ is the cardinality of the orbit of $v$ under the action of $\langle\alpha\rangle$ on $V(G)$.

THEOREM 4. If $\alpha \in$ Aut $G$, then $|F(\alpha)|=\prod_{v \in S}\left|F_{v}\left(\alpha^{l(v)}\right)\right|$, where the product extends over a complete set $S$ of orbit representatives for $\langle\alpha\rangle$ acting on $V(G)$.

PROOF.

$$
\begin{array}{lll}
\rho \in F(\alpha) & \text { if and only if } \quad \alpha \rho_{v} \alpha^{-1}=\rho_{\alpha(v)} \quad \forall v \in V(G), \\
& \text { if and only if } \alpha^{k} \rho_{v} \alpha^{-k}=\rho_{\alpha^{k}}(v) \quad \forall v \in V(G) \text { and } \forall k \in N .
\end{array}
$$

Thus, the rotation at $v$ determines the rotation at each vertex in the orbit of $v$ under $\alpha$. Moreover, when $k=l(v)$ we have $\alpha^{l(v)} \rho_{v} \alpha^{-l(v)}=\rho_{v}$, which implies $\rho_{v} \in F_{v}\left(\alpha^{l(v)}\right)$. Hence, if $S$ is a complete set of orbit representatives, the rotations on $G$ fixed by $\alpha$ are found by choosing $\rho_{v}$ in $F_{v}\left(\alpha^{l(v)}\right)$ for each $v \in S$. The rotations at the other vertices are then determined by $\rho_{\alpha^{k}(v)}=\alpha^{k} \rho_{v} \alpha^{-k}$. Since the choices on the orbits are independent the result follows.

To illustrate, consider the automorphism $\alpha=(12)(34)$ of $K_{4}$. Choose $S=\{1,3\}$ as the set of orbit representatives. Each orbit has length 2, so that $\left|F_{1}\left(\alpha^{l(1)}\right)\right|=$ $\left|F_{1}\left(\alpha^{2}\right)\right|=\mid F_{1}$ (identity) $\mid=2$, since there are two possible rotations at 1 , both fixed by the identity under conjugation. (Each of these forces the rotation of 2.) The same result follows for 3 and we have $|F(\alpha)|=2 \cdot 2=4$ as indicated in Table 1.

In the above example it happened that $\alpha^{l(v)}$ was the identity, so that $F_{v}\left(\alpha^{l(v)}\right)$ contained all the rotations at $v$. Although it cannot be expected that this is always the case, the next theorem shows that it is nevertheless not too difficult to determine $\left|F_{v}\left(\alpha^{l(v)}\right)\right|$ in general. In fact, the value is zero unless the permutation $\alpha^{l(v)}$ partitions the set of neighbors of $v$ into orbits having the same length.

In what follows, the cycle type of a permutation of an $n$-set is the $n$-tuple whose $k$ th entry is the number of $k$-cycles present in the disjoint cycle representation of 
the permutation. If $\gamma$ is the permutation, we write $j(\gamma)$ for the $n$-tuple and $j_{k}$ for the $k$ th entry. As usual, $\phi$ represents the Euler function and $N(v)$ the set of neighbors of the vertex $v$. Then $\left.\alpha^{l(v)}\right|_{N(v)}$ is the restriction of $\alpha^{l(v)}$ to the set of neighbors of $v$, and we let $n=|N(v)|$.

THEOREM 5 .

$$
\left|F_{v}\left(\alpha^{l(v)}\right)\right|=\left\{\begin{array}{l}
\phi(d)(n / d-1) ! d^{n / d-1} \\
\quad \text { if } j\left(\left.\alpha^{l(v)}\right|_{N(v)}\right)=\left(0, \ldots, 0, j_{d}=n / d, 0, \ldots, 0\right), \\
0 \text { otherwise. }
\end{array}\right.
$$

ProOF. To facilitate the proof let $X$ be $N(v), n=|X|$, and $\gamma$ the restriction of $\alpha^{l(v)}$ to $N(v)$. Since a member of $F_{v}\left(\alpha^{l(v)}\right)$ is a cyclic permutation of the neighbors of $v$ fixed by $\alpha^{l(v)}$ under conjugation, we want to determine those cyclic permutations of $X$ fixed by $\gamma$ under conjugation. If $\rho$ is an $n$-cycle such that $\gamma \rho \gamma^{-1}=\rho$, then $\gamma$ commutes with all powers of $\rho$. Let $\left(x_{1} x_{2} \cdots x_{n}\right)$ be the cycle representation of $\rho$ and let $x_{k+1}$ be the image of $x_{1}$ under $\gamma$. Then

$$
\gamma\left(x_{i+1}\right)=\gamma \rho^{i}\left(x_{1}\right)=\rho^{i} \gamma\left(x_{1}\right)=\rho^{i}\left(x_{k+1}\right)=\rho^{i} \rho^{k}\left(x_{1}\right)=\rho^{k} \rho^{i}\left(x_{1}\right)=\rho^{k}\left(x_{i+1}\right),
$$

$i=0,1, \ldots, n-1$, so that $\gamma=\rho^{k}$. Since the $k$ th power of an $n$-cycle has cycle type $\left(0, \ldots, 0, j_{d}=n / d, 0, \ldots, 0\right)$ where $d=n / \operatorname{gcd}(k, n), \gamma$ must have this cycle type, else it fixes no $n$-cycle under conjugation.

To determine the number of $n$-cycles fixed by a permutation of the above type, let $J$ be the set of $n ! /\left(d^{n / d}(n / d) !\right)$ permutations of $X$ with cycle type $\left(0, \ldots, 0, j_{d}=\right.$ $n / d, 0, \ldots, 0)$ and $C$ the set of $(n-1)$ ! cyclic permutations of $X$. For each $\gamma \in J$, let $C_{\gamma}$ denote those members of $C$ fixed by $\gamma$ under conjugation and note that no element of $J$ is distinguished over any other, so that each $C_{\gamma}$ has the same cardinality, the number desired. Denote this common value by $c$; then

$$
\sum_{\gamma \in J}\left|C_{\gamma}\right|=c \cdot|J|
$$

It will be shown that each $n$-cycle of $C$ is fixed under conjugation by precisely $\phi(d)$ members of $J$, and hence

$$
\sum_{\gamma \in J}\left|C_{\gamma}\right|=\phi(d) \cdot|C|
$$

in which case

$$
c=\frac{\phi(d) \cdot|C|}{|J|}=\frac{\phi(d)(n-1) !}{n ! /\left(d^{n / d}(n / d) !\right)},
$$

which simplifies to the desired result. To this end recall that $\gamma$ in $J$ fixes $\rho$ in $C$ implies $\gamma$ is a $k$ th power of $\rho$, where $k$ satisfies $\operatorname{gcd}(k, n)=n / d$ (and clearly, these powers of $\rho$ fix $\rho$ under conjugation). Thus, we need to determine the number of integers $k$ such that $\operatorname{gcd}(k, n)=n / d$ where $1 \leq k \leq n$. The solutions are given by $k=m \cdot(n / d)$ where $m$ and $d$ are relatively prime and $1 \leq m<d$. Thus, there are $\phi(d)$ solutions.

Returning to the example, consider the automorphism $\alpha=(123)(4)$ of $K_{4}$. Choose $S=\{1,4\}$ as the set of orbit representatives. Let $e$ be the identity of $S_{4}$; then by Theorem 4 we have

$$
|F(\alpha)|=\left|F_{1}\left(\alpha^{3}\right)\right| \cdot\left|F_{4}\left(\alpha^{1}\right)\right|=\left|F_{1}(e)\right| \cdot\left|F_{4}(\alpha)\right|=2\left|F_{4}(\alpha)\right| .
$$


Since $\left.\alpha^{l(4)}\right|_{N(v)}=(123)$ has cycle type $\left(0,0, j_{3}=3 / 3\right)$, we have by Theorem 5 that

$$
\left|F_{4}(\alpha)\right|=\phi(3)(3 / 3-1) ! 3^{3 / 3-1}=2 .
$$

Hence, $|F(\alpha)|=2 \cdot 2=4$, as shown in Table 1 .

4. Applications of the general theory. To illustrate the use of these results, we first consider that class of graphs known as wheels. A wheel on $n+1$ vertices, $W_{n+1}$, consists of an $n$-cycle of vertices, each joined to a central vertex. If $n \geq 4$, then Aut $W_{n+1}$ is the dihedral group $D_{n}$ of order $2 n$.

Let $v$ be the central vertex of $W_{n+1}, n \geq 4$, and $\alpha \in$ Aut $W_{n+1}$. Then $l(v)=$ 1 , and by Theorem 5 we have that $\left|F_{v}\left(\alpha^{1}\right)\right| \neq 0$ if and only if $j\left(\left.\alpha\right|_{N(v)}\right)=$ $\left(0, \ldots, 0, j_{d}=|N(v)| / d, 0, \ldots, 0\right)$, for some divisor $d$ of $|N(v)|$. Since $|N(v)|=n$, it follows from the above and Theorem 5 that

$$
\left|F_{v}(\alpha)\right|=\phi(d) \cdot((n / d)-1) ! d^{n / d-1}
$$

if $j(\alpha)=\left(1,0, \ldots, 0, j_{d}=n / d, 0, \ldots, 0\right)$, and is zero otherwise. If $u$ is any other vertex, then $l(u)=d$, so that $\left|F_{u}\left(\alpha^{d}\right)\right|=\left|F_{u}(e)\right|$. Since the identity permutation fixes all of the (degree $(u)-1)$ ! rotations at $u$ and the degree of $u$ is 3 , we have $\left|F_{u}\left(\alpha^{l(u)}\right)\right|=2$. Thus $|F(\alpha)| \neq 0$ if and only if $\alpha$ has the above cycle type, in which case, by Theorem 4 ,

$$
|F(\alpha)|=2^{n / d} \phi(d)((n / d)-1) ! d^{n / d-1} .
$$

The number of permutations in Aut $W_{n+1}(n \geq 4)$ with cycle type $\left(1,0, \ldots, 0, j_{d}\right.$ $=n / d, 0, \ldots, 0)$ is $\phi(d)$ if $d$ is not 2 (corresponding to the rotations of a regular $n$ gon) and $1+n / 2$ if $d$ equals 2 with $n$ even (the $180^{\circ}$ rotation and the $n / 2$ reflections of a regular $n$-gon leaving no vertex fixed). Thus, we have the following result, by Theorem 2:

THEOREM 6. For $n \geq 4$,

$$
\left|C\left(W_{n+1}\right)\right|=\left\{\begin{array}{l}
\frac{1}{2 n} \sum_{d \mid n} \phi^{2}(d) \cdot 2^{n / d}\left(\frac{n}{d}-1\right) ! d^{n / d-1}, \quad n \text { odd } \\
\frac{1}{2 n} \sum_{d \mid n} \phi^{2}(d) \cdot 2^{n / d}\left(\frac{n}{d}-1\right) ! d^{n / d-1}+2^{n-3}\left(\frac{n}{2}-1\right) !, \quad n \text { even. }
\end{array}\right.
$$

In Table 2, we give the first few values, for this counting function.

TABLE 2

$$
\begin{array}{ccccccc}
n & 4 & 5 & 6 & 7 & 8 & 9 \\
\left|C\left(W_{n+1}\right)\right| & 16 & 80 & 666 & 6,588 & 80,886 & 1,146,916
\end{array}
$$

Now consider the complete graph on $n$ vertices, $K_{n}$. The automorphism group of $K_{n}$ is the full symmetric group $S_{n}$. Since any two distinct vertices of $K_{n}$ are adjacent, it follows from Theorem 3 that only those automorphisms which fix 0,1 , or all of the vertices need to be considered.

If $\alpha$ fixes none of the vertices, then $l(v) \geq 2$ for each vertex $v$, and by Theorem $4|F(\alpha)| \neq 0$ if and only if $\left|F_{v}\left(\alpha^{l(v)}\right)\right| \neq 0$, for all $v \in V\left(K_{n}\right)$, if and only if $j\left(\left.\alpha^{l(v)}\right|_{N(v)}\right)=\left(0, \ldots, 0, j_{k}=|N(v)| / k, 0, \ldots, 0\right)$, for all $v \in V\left(K_{n}\right)$-by Theorem 5 , and where $k$ depends on $v$. Since $|N(v)|=n-1$ and $\left.\alpha^{l(v)}\right|_{N(v)}$ must fix at least one vertex (those in the orbit of $v$ under $\alpha$ ), it follows that $k=1$ and, thus, 
$\alpha^{l(v)}$ is the identity permutation on $N(v)$ for each vertex $v$. This is only possible if $j(\alpha)=\left(0, \ldots, 0, j_{d}=n / d, 0, \ldots, 0\right)$ for some divisor $d$ of $n$, in which case

$$
\left|F_{v}\left(\alpha^{l(v)}\right)\right|=\left|F_{v}(e)\right|=(\operatorname{degree}(v)-1) !=(n-2) !,
$$

and $|F(\alpha)|=(n-2) !^{n / d}$. The number of nonidentity permutations in $S_{n}$ with this cycle type is $n ! /\left(d^{n / d}(n / d) !\right)$, for each divisor $d$ of $n$. The contribution to $\left|C\left(K_{n}\right)\right|$ made by all such permutation is

$$
\frac{1}{n !} \sum_{\substack{d \mid n \\ d \neq 1}} \frac{n !}{d^{n / d}(n / d) !}(n-2) !^{n / d}
$$

Since the identity permutation fixes all $(n-2) !^{n}$ rotations, the above sum can be extended to include $d=1$. It remains to determine the contribution from those automorphisms which fix exactly one vertex.

If $\alpha$ fixes exactly one vertex, say $v$, then by Theorem $4|F(\alpha)| \neq 0$ implies $\left|F_{v}(\alpha)\right| \neq 0$ if and only if $j\left(\left.\alpha\right|_{N(v)}\right)=\left(0, \ldots, 0, j_{d}=(n-1) / d, 0, \ldots, 0\right)$ if and only if $j(\alpha)=\left(1,0, \ldots, 0, j_{d}=(n-1) / d, 0, \ldots, 0\right)$ for some divisor $d$ of $n-1$. If this is the case, we have, from Theorem 5 , that

$$
\left|F_{v}(\alpha)\right|=\phi(d)((n-1) / d-1) ! d^{(n-1) / d-1} .
$$

If $u$ is any vertex other than $v$, then

$$
\left|F_{u}\left(\alpha^{l(u)}\right)\right|=\left|F_{u}\left(\alpha^{d}\right)\right|=\left|F_{u}(e)\right|=(n-2) !,
$$

and it follows that

$$
|F(\alpha)|=(n-2) !^{(n-1) / d} \phi(d)((n-1) / d-1) ! d^{(n-1) / d-1},
$$

provided $\alpha$ has the above cycle type. The number of automorphisms of this type in $S_{n}$ is $n ! /\left(d^{(n-1) / d}((n-1) / d)\right.$ !, for each divisor $d$ of $n-1$. The contribution to $\left|C\left(K_{n}\right)\right|$ made by all those permutations which fix exactly one vertex is then

$$
\frac{1}{n !} \sum_{\substack{d \mid(n-1) \\ d \neq 1}} \frac{n !}{d^{(n-1) / d}((n-1) / d) !}(n-2) !^{(n-1) / d} \phi(d)\left(\frac{n-1}{d}-1\right) ! d^{(n-1) / d-1} .
$$

Performing a few simplifications, we present the following counting theorem

THEOREM 7 .

$$
\left|C\left(K_{n}\right)\right|=\sum_{d \mid n} \frac{(n-2) !^{n / d}}{d^{n / d}(n / d) !}+\sum_{\substack{d \mid(n-1) \\ d \neq 1}} \frac{\phi(d)(n-2) !^{(n-1) / d}}{n-1} .
$$

In Table 3, we give the first few values of this function.

TABLE 3

$$
\begin{array}{ccccccc}
n & 2 & 3 & 4 & 5 & 6 & 7 \\
\left|C\left(K_{n}\right)\right| & 1 & 1 & 3 & 78 & 265,764 & 71,095,150,000
\end{array}
$$

5. Asymptotic results. We note that the numbers given in the previous section grow large quickly. In this section we see just how quickly by giving the asymptotic results. 
THEOREM 8.

$$
\operatorname{Lim}_{n \rightarrow \infty} \frac{\left|C\left(K_{n}\right)\right|}{(n-2) !^{n} / n !}=1
$$

PROOF. Clearly

$$
\lim _{n \rightarrow \infty} \frac{\left|C\left(K_{n}\right)\right|}{(n-2) !^{n} / n !} \geq 1,
$$

as the first term of $\left|C\left(K_{n}\right)\right|$, corresponding to $d=1$, is just $(n-2) !^{n} / n !$. Thus we calculate

$$
\begin{aligned}
& \lim _{n \rightarrow \infty} \frac{\left|C\left(K_{n}\right)\right|}{(n-2) !^{n} / n !} \\
& =\lim _{n \rightarrow \infty} \frac{n !}{(n-2) ! n}\left(\sum_{d \mid n} \frac{(n-2) !^{n / d}}{d^{n / d}(n / d) !}+\sum_{\substack{d \mid(n-1) \\
d \neq 1}} \frac{\phi(d)(n-2) !^{(n-2) / d}}{n-1}\right) \\
& =\lim _{n \rightarrow \infty}\left(1+\sum_{\substack{d \mid n \\
d \neq 1}} \frac{n(n-1)(n-2) !^{(n+d) / d}}{d^{n / d}(n / d) !(n-2) !^{n}}+\sum_{\substack{d \mid(n-1) \\
d \neq 1}} \frac{n \phi(d)(n-2) !^{(n+d-1) / d}}{(n-2) !^{n}}\right) .
\end{aligned}
$$

Now $\sum_{d \mid n} \phi(d)=m$ for every positive integer $m$ (see Rosen [4], for example); thus we can estimate

$$
\lim _{n \rightarrow \infty} \frac{\left|C\left(K_{n}\right)\right|}{(n-2) !^{n} / n !} \leq 1+\lim _{n \rightarrow \infty}\left(\frac{n(n-1) !}{2(n-2) !^{(n-2) / 2}}+\frac{n(n-1)}{(n-2) !^{(n-1) / 2}}\right) .
$$

In both fractions the denominators will clearly dominate, and the result follows.

COROLlaRY 9. Asymptotically, $\left|C\left(K_{n}\right)\right|=\left|R\left(K_{n}\right)\right| / \mid$ Aut $K_{n} \mid$.

A similar analysis establishes

THEOREM 10.

$$
\operatorname{Lim}_{n \rightarrow \infty} \frac{\left|C\left(W_{n+1}\right)\right|}{2^{n}(n-1) ! / 2 n}=1 .
$$

COROLlaRY 11. Asymptotically,

$$
\left|C\left(W_{n+1}\right)\right|=\left|R\left(W_{n+1}\right)\right| / \mid \text { Aut } W_{n+1} \mid .
$$

6. Problems. The noteworthy feature about the results of the last section is that we need only consider the identity automorphism to get a fairly good idea of the number of congruence classes, for $n$ large, if we are considering $K_{n}$ or $W_{n+1}$. Other families of graphs may not behave this way, as the $n$-cycles $C_{n}$ readily demonstrate $\left(\left|R\left(C_{n}\right)\right|=1, \mid\right.$ Aut $\left.C_{n}|=2 n| C,\left(C_{n}\right) \mid=1\right)$.

This suggests the problem of characterizing those classes of graphs $G_{n}$ of order $n$ having the property that, for $n$ sufficiently large, $\left|C\left(G_{n}\right)\right| \approx\left|R\left(G_{n}\right)\right| / \mid$ Aut $G_{n} \mid$.

Finally, we propose the following enumerating polynomials for further study. (See Gross and Furst [6], where the corresponding polynomials $g[G](x)$ and $i[G]\left(z_{j}\right)$ prior to the application of the equivalence relation under study here are introduced.) The congruence class genus polynomial for a connected graph $G$, denoted $C g[G](x)$, 
counts the number of congruence classes of 2-cell imbeddings on each orientable surface: the coefficient of $x^{k}$ gives this number for the surface of genus $k$. For example:

$$
\begin{aligned}
g\left[K_{4}\right](x) & =2+14 x \\
C g\left[K_{4}\right](x) & =1+2 x \\
g\left[K_{5}\right](x) & =462 x+4974 x^{2}+2340 x^{3} \\
C g\left[K_{5}\right](x) & =9 x+45 x^{2}+24 x^{3} .
\end{aligned}
$$

The coefficients of the congruence class imbedding polynomial give the number of congruence classes for maps having a given region distribution. For a given graph $G$, we denote this by $C i[G]\left(z_{j}\right)$. The exponent on variable $z_{j}$ gives the number of regions of the imbedding bounded by $j$ edges. We have no sophisticated method of computing these coefficients. However, for example, hand and computer analyses yield:

$$
\begin{aligned}
i\left[K_{4}\right]\left(z_{j}\right)= & 2 z_{3}^{4}+8 z_{3} z_{9}+6 z_{4} z_{8} \\
C i\left[K_{4}\right]\left(z_{j}\right)= & z_{3}^{4}+z_{3} z_{9}+z_{4} z_{8} \\
i\left[K_{5}\right]\left(z_{j}\right)= & 150 z_{3}^{4} z_{8}+120 z_{3}^{3} z_{4} z_{7}+120 z_{3}^{2} z_{4} z_{6}+60 z_{3}^{2} z_{4} z_{5}+12 z_{4}^{5} \\
& +960 z_{3}^{2} z_{14}+960 z_{3} z_{4} z_{13}+240 z_{3} z_{5} z_{12}+240 z_{3} z_{6} z_{11} \\
& +360 z_{3} z_{7} z_{10}+720 z_{3} z_{8} z_{9}+240 z_{4}^{2} z_{12}+120 z_{4} z_{5} z_{11} \\
& +420 z_{4} z_{6} z_{10}+360 z_{4} z_{7} z_{9}+60 z_{4} z_{8}^{2}+24 z_{5}^{2} z_{10} \\
& +120 z_{5} z_{6} z_{9}+120 z_{5} z_{7} z_{8}+30 z_{6}^{2} z_{8}+2340 z_{20} \\
C i\left[K_{5}\right]\left(z_{j}\right)= & 3 z_{3}^{4} z_{8}+z_{3}^{3} z_{4} z_{7}+2 z_{3}^{2} z_{4}^{2} z_{6}+z_{3}^{2} z_{4} z_{5}^{2}+2 z_{4}^{5} \\
& +8 z_{3}^{2} z_{14}+8 z_{3} z_{4} z_{13}+2 z_{3} z_{5} z_{12}+2 z_{3} z_{6} z_{11} \\
& +3 z_{3} z_{7} z_{10}+6 z_{3} z_{8} z_{9}+2 z_{4}^{2} z_{12}+z_{4} z_{5} z_{11} \\
& +4 z_{4} z_{6} z_{10}+3 z_{4} z_{7} z_{9}+2 z_{4} z_{8}^{2}+z_{5}^{2} z_{10} \\
& +z_{5} z_{6} z_{9}+z_{5} z_{7} z_{8}+z_{6}^{2} z_{8}+24 z_{20} .
\end{aligned}
$$

To aid in the interpretation of these polynomials, we continue with the $K_{4}$ example. From $G\left[K_{4}\right](x)=2+14 x$, we see that two of the sixteen rotations for $K_{4}$ are spherical; the other fourteen are toroidal. From $C g\left[K_{4}\right](x)=1+2 x$, we see that the two spherical rotations form a single congruence class, while the fourteen toroidal rotations split into two classes. From $i\left[K_{4}\right]\left(z_{j}\right)=2 z_{3}^{4}+8 z_{3} z_{9}+6 z_{4} z_{8}$, we find that the two spherical rotations are both triangulations, while the fourteen toroidal rotations split into eight imbeddings having one triangle and one nonagon each, and six imbeddings having one quadrilateral and one octagon each. From $C i\left[K_{4}\right]\left(z_{j}\right)=z_{3}^{4}+z_{3} z_{9}+z_{4} z_{8}$, we see that these three region distributions determine one congruence class each.

In general, we note that $g[G](1)=i[G](1,1, \ldots, 1)=|R(G)|$, and that $C g[G](1)$ $=C i[G](1,1, \ldots, 1)=|C(G)|$. Here, we check that $\left|R\left(K_{4}\right)\right|=16,\left|C\left(K_{4}\right)\right|=3$. We also verify that $\left|R\left(K_{5}\right)\right|=7776,\left|C\left(K_{5}\right)\right|=78$.

ACKNOWLEDGEMENT. We thank the referee for many helpful comments and suggestions. 


\section{REFERENCES}

1. N. L. Biggs, Automorphisms of imbedded graphs, J. Combin. Theory B 11 (1971), 132-138.

2. N. L. Biggs and A. T. White, Permutation groups and combinatorial structures, Cambridge Univ. Press, Cambridge, 1979.

3. J. L. Gross and M. Furst, Hierarchy for imbedding-distribution invariants of a graph, J. Graph Theory 11 (1987), 205-220.

4. K. H. Rosen, Elementary number theory and its applications, Addison-Wesley, Reading, Mass., 1974.

5. A. T. White, Graphs, groups and surfaces, rev. ed., North-Holland, Amsterdam, 1984.

Department of Mathematics, Western Michigan University, Kalamazoo, MiCHIGAN 49008 (Current addresss of B. P. Mull and A. T. White)

Current address (R. G. Rieper): Department of Computer Science, Columbia University, New York, New York 10027 